\title{
Analysis on the Coupling Type Optoelectronic Oscillator and its Application
}

\author{
Jianfang $\mathrm{Li}^{1, \mathrm{a}}$ \\ ${ }^{1}$ Chongqing College of Electronic Engineering, Chongqing 401331, China \\ alijianfang023023@126.com
}

Keywords: Coupling type, Optoelectronic oscillator, Microwave signal

\begin{abstract}
The basic structure of the optoelectronic oscillator is based on photoelectric hybrid feedback loop; the optical signal is converted into the microwave signal directly. Laser as a power source, is a continuous light. Laser through the electro-optic modulator, optical fiber delay line and photoelectric is converted into the microwave signal. Microwave signal through amplifier, filter, and the part of coupler is output, and another part of the feedback back to the electro-optic modulator with modulated light waves. The output signal frequency of the optoelectronic oscillator is determined by the microwave filter. Therefore, the frequency source becomes the signal receiver frequency drift whether a key link in the process of phase jitter, waveform distortion, etc. This paper will discuss the coupling type optoelectronic oscillator and its application.
\end{abstract}

\section{Introduction}

The oscillator is a communication system, navigation, radar, instrument, electronic system of key devices, such as the electronic countermeasure, a direct impact on the performance of electronic systems. Oscillator can provide the clock signal for the high-speed digital system, as the implementation of vibration, frequency conversion, as a reference source in the synchronization system. The development of modern communication technology is to high bandwidth, high frequency; further improve the performance of microwave oscillator. With the development of monolithic microwave integrated circuits, various types of transistor oscillator has been widely used in various fields, become the mainstream of the microwave oscillator today. In the $1990 \mathrm{~s}$, a new type of microwave oscillator using photoelectric feedback loop technology, optoelectronic oscillator (optoelectronic oscillator, OEO), in the microwave/millimeter wave band shows unique advantages, the phase noise is much better than the traditional microwave oscillator.

\section{The Working Principle and Characteristics of the Optoelectronic Oscillator}

The optoelectronic oscillator is generally consists of light source, intensity modulator, filter, photoelectric detector (PD) consisting of a positive feedback loop. It USES modulator and the characteristics of optical fiber low loss will be continuous light into a stable, clean $\mathrm{rf} /$ microwave spectrum signal, its structure is shown in figure 1. Continuous from the laser light through the optical fiber transmission after electro-optic modulator in the photoelectric detector, photoelectric detector convert light into electrical signals in the selected frequency modulator, amplification and feedback. In the process of the active device will produce different frequency noise disturbance, the disturbance on the output side are filter out hope that the vibration frequency, and used for feedback control of electro-optic modulator. Loop of the amplifier provides gain and signal, after many cycles can establish a stable oscillation; the oscillation frequency is mainly determined by the filter pass band characteristic. Through theoretical analysis and experiment, loop energy decay time of energy storage capacity (medium) determines the $\mathrm{Q}$ value of the oscillator. There is $Q=2 \pi f t_{d}$, and $f$ is the oscillation frequency, $t_{d}$ is the energy decay time. Therefore, as long as the loop with enough length of optical fiber, optoelectronic oscillator can produce higher quality than traditional oscillator signal output. In addition, the study also points out that the photoelectric oscillator phase noise with the amount of deviation $f^{\prime}$ is a quadratic drop, and for fixed $f^{\prime}$, Delay is a quadratic 
phase noise with loop drops and has nothing to do with the work frequency of the oscillator. While phase noise is not infinite decreases with the increase of the loop delay [SNR finally under the control of the relative intensity noise (RIN)] of laser, but the use of long fiber is still an inevitable trend.

The optoelectronic oscillator performance is outstanding, but it also determines the system structure of some of its shortcomings. First, in order to get high Q value of the output signal, general need long optical fiber used in cavity, while the length of cavity length also determines the vibration mode between the interval, the longer the cavity, the model of interval. Can theoretically with a sufficiently narrow filter out unwanted pattern, but on the device to obtain considerable difficulties; next, the phase noise of the signal and, light relative intensity noise, photoelectric sensors and power amplifier will be the final phase noise of microwave signal generated by the impact. Filter, amplifier bandwidth is too large will make pass band range. The signal-to-noise ratio is reduced, affect the vibration frequency of quality; Finally, because the loop is made up of optical fiber, and its cavity length is vulnerable to environment change the influence of temperature, stress, etc, the fundamental frequency of vibration caused by the change of the output or frequency hopping frequency drift; In addition, long fiber occupies larger volume, miniaturization, integration of the photoelectric generator system. To solve these problems are some of the key optoelectronic oscillator the practical work.

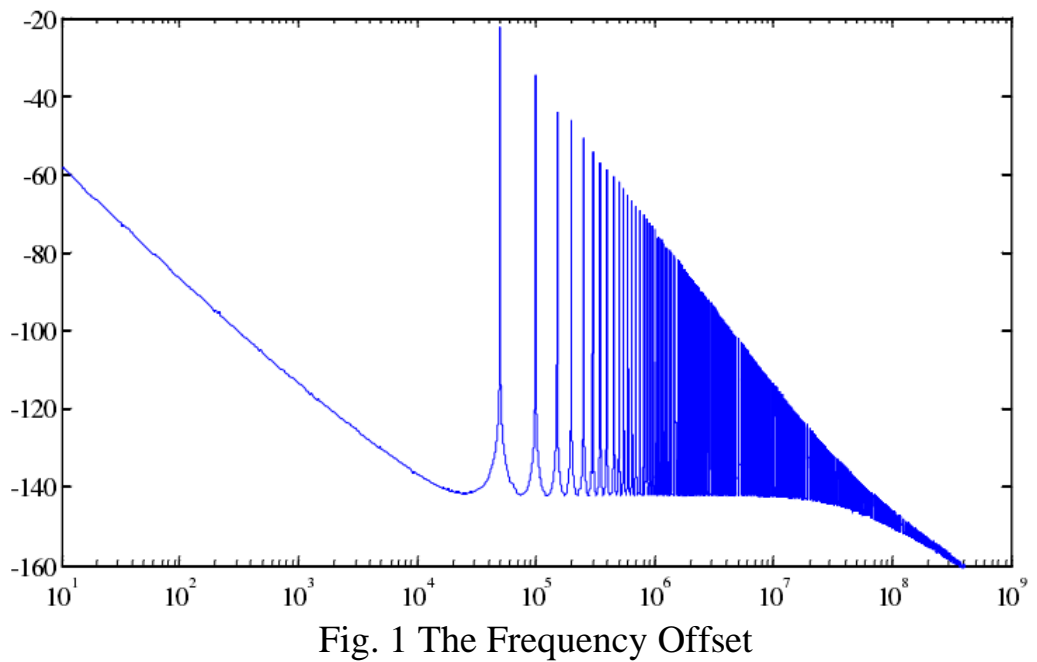

\section{The Frequency Stability}

How to improve the frequency stability is one of the key OEO practical. Factors affecting the stability of OEO effective length caused by the external environment change, laser polarization of ups and downs in the optical fiber, as well as the boundary between the die and the main die gain competition, etc. By inhibiting the edge mould, can greatly improve the stability of OEO. Because of laser polarization of the movement, temperature and fiber bending are particularly sensitive to the change of polarization can actually lead to the effective cavity length change, so that the oscillation frequency drift. In order to eliminate the effects of laser polarization ups and downs, all risks polarizing device can be used, but it will also increase the cost of OEO. In essence, to enhance the stability of OEO, it is necessary to the effective cavity length control OEO; reduce the influence of the external environment. The optoelectronic integrated is the inevitable development trend of high-speed semiconductor optoelectronic devices technology, was the key to OEO can truly miniaturization and commercialization. Optoelectronic integration is to integrate the photoelectric material and function of microstructure on the single chip, to achieve a variety of functions. For OEO, need is the photoelectron discrete device, such as laser, electro-optic modulator, optical fiber (or the energy storage unit), photoelectric detector, microwave filter (or photons), etc., through reasonable optimization, design, processing and packaging technology, integrated into a single chip. 


\section{The Inhibition of the Phase Noise}

The phase noise is a key indicator to evaluate the quality of the microwave signal. In the system of optoelectronic oscillator, the output signal's phase noise mainly comes from the photoelectric detector, amplifier and some active components of the laser scattering noise, thermal noise, relative intensity noise. And already mentioned before, the use of long optical fiber phase noise can be reduced effectively, but not infinite. Therefore, a direct method to reduce phase noise is the introduction of high-performance devices used to reduce noise, such as using a high $\mathrm{Q}$ value filter can reduce the noise in the zone; High quality amplifier, light also can improve the photoelectric oscillator noise performance. In $143 \mathrm{DBC} / \mathrm{Hz}$ low phase noise signal Experiments, is used with very low relative intensity noise of Nd: YAG laser. In addition, under the condition of a specific device, the researchers also proposed some by optimizing the photonic microwave link and changed the way devices work methods to reduce optoelectronic oscillator phase noise. Danny Eliyahu, by studying the relative intensity of the laser and microwave signal noise, points out that the transformation of the relationship between use of saturated working state of the active device can inhibit the relative intensity noise and improve the performance of optoelectronic oscillator. Theory and experiment show that the optoelectronic oscillator circuits to insert a semiconductor optical amplifier (SOA) and make it work in saturated state (SGM) using its self gain modulation effect, to about $10 \mathrm{db}$ relative intensity noise suppression. In addition, if the photo detector is working in a saturated state, it can significantly improve the quality of output microwave signals. For electricity amplifier introduced in the system $1 / f$ noise, can use multiple photoelectric detector light signal and carrier suppression technology is adopted to suppress. Carrier suppression technology to match the multiple loop structure, in inhibition of phase noise at the same time also can constitute a phase-locked structure in order to improve the output optoelectronic oscillator frequency stability.

Factors influencing the output optoelectronic oscillator frequency stability has two aspects: one is the cavity length is affected by environment change, make up the model of vibration changes destabilize the output frequency; 2 it is because the filter used in the optoelectronic oscillator system usually has larger scope of pass band, so within the loop gain bandwidth will appear very multilateral mode. One of these edge moulds could be obtained in the process of cavity length change gain enough to replace the original frequency, causing the vibration frequency of instability.

The phase-locked loop is a kind of commonly used to improve the stability of the system frequency; it is also conducive to optoelectronic oscillator frequency stability of the output. It is introduced in the multiple loop structure through carrier suppression technology of phase locking scheme, this scheme as a whole and to achieve the edge mode rejection of optoelectronic oscillator output signal, to reduce the phase noise and frequency stability, the principle of phase lock part as shown in Fig. 2.

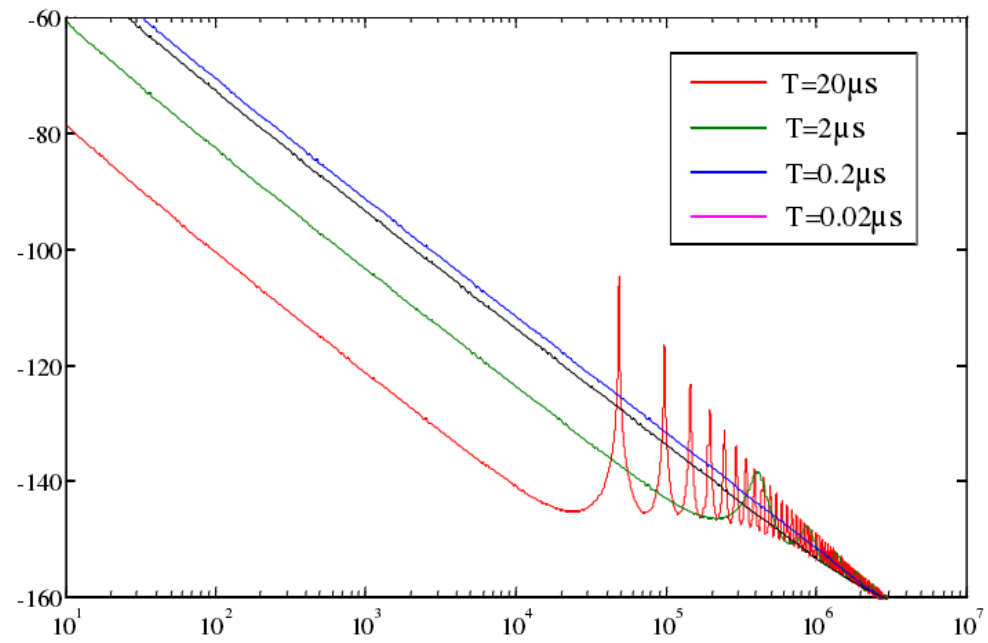

Fig. 2 The Microwave Optoelectronic Oscillator Phase Noise Spectrum

The oscillator after long light output all the way through the optical fiber transmission delay is photo detector is converted to electrical signal as reference signal, and then with all the oscillator 
output signal coupling. Carefully adjust the intensity of the signal and phase allows two-way coupling output all the way for the suppression of output (when the coupling of two signal phase difference, all the way for the carrier to enhance (coupling of two signals in phase). The carrier can inhibit the output carrier by inhibition of about $40 \mathrm{~dB}$, carrier to noise ratio increases, the process can make the frequency (phase) jitter into the change of amplitude, and the signal into the amplifier amplification, the amplifier will not saturated. Be amplified signal to a power output of oscillator signal mixer (vibration), including the frequency output role in voltage controlled phase shifter to adjust the phase of the carrier to enhance all the way, then feedback modulation. In such a structure, meet the best carrier suppression condition, in the oscillation circuit can realize the maximum gain, therefore, this scheme can guarantee the output of the oscillator can track length change, maintain the stability of a few hours work. At the same time due to the elimination of frequency jitter, phase noise performance has improved, and multiple loop for side mode suppression characteristics also got play, the experimental results show that the $10 \mathrm{~Hz}$ from the carrier phase noise gets about 20 $\mathrm{dB}$.

Through a low frequency signal injection locking of higher harmonic, also can be regarded as a method of stable high frequency output. Using a stable low frequency signal modulation nonlinear or other nonlinear effect of optical fiber after injection of optoelectronic oscillator, high-order harmonic generation (HHG in optoelectronic oscillator to choose suitable filter lock in a high frequency harmonic signal feedback, you can generate a high frequency signal output, high quality and have good frequency stability, The research demonstrates the experiment scheme, etc. Optoelectronic oscillator's basic function is to produce high quality, light, electricity and microwave signals, but through the development, also derive some new way of application. In these applications, the microwave power output part of the basic signal optoelectronic oscillator output, but the light output part there has been some changes.

\section{Summary}

The optoelectronic oscillator as a kind of high quality light, electricity microwave signal generator has great advantages and widely applicable, various applications of distinctive means for optoelectronic oscillator multi-functional laid a good foundation. But there is no denying that the optoelectronic oscillator is mainly at the laboratory research stage, to make it in the national economic construction and national defense science and technology development in practical application is still a process. The main restricting factors on how to put the optoelectronic oscillator system into a compact miniaturization, integration, facilitate the frequency control system, the implementation of these requirements will depend on the development of new photonic microwave devices and corresponding active device and further perfect craftsmanship.

\section{References}

[1] Badami V G,Patterson S R.A frequency domain method for the measurement of nonlinearity in heterodyne interferometry. Precision Engineering. 2011(08).

[2] Hayashi I,Panish MB,Foy PW,et al.Junction lasers which operate continuously at room temperature. Applied Physics. 2012(10).

[3] Kapron FP,Keck DB,Mauer RD.Radiation loss in glass optical waveguides. Applied Physics. 2013(11).

[4] Mears R J,Reekie L,Jauncey I M,et al.Low-noise erbium-doped fiber amplifier operation at 1.54 um. Electronics Letters. 2011(11).

[5] Desurvire E,Simpson JR,Becker PC.High-gain erbium-doped traveling-wave fiber amplifier. Optics Letters. 2012(10). 